\title{
Development of spatial integration depends on top-down and interhemispheric connections that can be perturbed in migraine: a DCM analysis
}

\author{
Eleonora Fornari • Romana Rytsar • \\ Maria G. Knyazeva
}

(C) Springer-Verlag Italia 2014

\begin{abstract}
In humans, spatial integration develops slowly, continuing through childhood into adolescence. On the assumption that this protracted course depends on the formation of networks with slowly developing top-down connections, we compared effective connectivity in the visual cortex between 13 children (age 7-13) and 14 adults (age 21-42) using a passive perceptual task. The subjects were scanned while viewing bilateral gratings, which either obeyed Gestalt grouping rules [colinear gratings (CG)] or violated them [non-colinear gratings (NG)]. The regions of interest for dynamic causal modeling were determined from activations in functional MRI contrasts stimuli $>$ background and CG $>$ NG. They were symmetrically located in $\mathrm{V} 1$ and $\mathrm{V} 3 \mathrm{~V}$ areas of both hemispheres. We studied a common model, which contained reciprocal intrinsic and modulatory connections between these
\end{abstract}

E. Fornari and R. Rytsar contributed equally to this work.

\section{E. Fornari}

CHUV Unit, CIBM (Centre d'Imagérie Biomédicale), Centre Hospitalier Universitaire Vaudois (CHUV), and University of Lausanne, Rue du Bugnon, 46, 1011 Lausanne, Switzerland

E. Fornari $(\bowtie) \cdot$ M. G. Knyazeva

Department of Radiology, Centre Hospitalier Universitaire Vaudois (CHUV), and University of Lausanne, Rue du Bugnon, 46, 1011 Lausanne, Switzerland e-mail: Eleonora.Fornari@chuv.ch

M. G. Knyazeva

e-mail: Maria.Knyazeva@chuv.ch

R. Rytsar · M. G. Knyazeva

Department of Clinical Neuroscience, Centre Hospitalier

Universitaire Vaudois (CHUV), and University of Lausanne,

Rue du Bugnon, 46, 1011 Lausanne, Switzerland

e-mail: Romana.Rytsar@gmail.com regions. An analysis of effective connectivity showed that top-down modulatory effects generated at an extrastriate level and interhemispheric modulatory effects between primary visual areas (all inhibitory) are significantly weaker in children than in adults, suggesting that the formation of feedback and interhemispheric effective connections continues into adolescence. These results are consistent with a model in which spatial integration at an extrastriate level results in top-down messages to the primary visual areas, where they are supplemented by lateral (interhemispheric) messages, making perceptual encoding more efficient and less redundant. Abnormal formation of top-down inhibitory connections can lead to the reduction of habituation observed in migraine patients.

Keywords Children · Effective connectivity - Inhibition · Predictive coding · Visual cortex · Functional magnetic resonance imaging

\section{Introduction}

The developing brain provides a unique opportunity to investigate its functional networks in consecutive temporal windows. Due to the interregional heterochronicity of maturation, the role of long-developing functional circuits and their dysfunction in pathology can be understood. The top-down influences are based on such circuits with protracted development $[1,2]$. Their extended development through adolescence has been shown for prefrontal topdown influences underlying voluntary behavioral control $[3,4]$. However, top-down effects are involved in all types of cognitive activities, including perception, where they reshape interactions between distributed networks under different perceptual contexts. 
Moreover, their deviant or delayed development might be suspected in the pathological conditions that affect distributed brain regions and have age-dependent prevalence. For instance, migraine, which is a frequent cause of headache in children, can be among such neurological disorders. Indeed, as MRI-based studies in adults show distributed cerebral networks are changed in chronic migraine patients $[5,6]$. The prevalence of migraine increases from 3 to $19 \%$ in children between 5 and 12-year-old, and then drops to some extent [7], suggestive of a link to certain protracted developmental processes.

The predictive coding hypothesis [8] persuasively offers a framework for the role of top-down effects in the hierarchical processing of sensory stimuli. This hypothesis suggests that at each level of signal processing, neural networks form internal models of the natural world based on its regularities. These representations generate predictions of how the sensory evidence should be interpreted and send them via descending connections upstream, where the model information is compared to the stimulus-driven activity. At the lower processing level, the well-predicted activity is removed by inhibition, and the residual error between the prediction and sensory evidence is calculated. The signal deviations from the predictions are sent to the higher processing centers for adjusting the model. Importantly, by inhibiting the predictable and, therefore, excessive activity, such a hierarchical mechanism optimizes processing of a stimulus.

Top-down influences can be studied non-invasively in terms of effective connectivity, which evaluates the influence that one local neural system (source) exerts on another (target) by means of dynamic causal modeling (DCM) [9]. For the analysis of feedback effects, it is important that DCM differentiates positive and negative couplings. Positive modulation expresses functional excitation in a target region in the case of activity increase correlated with the source, while negative modulation manifests functional inhibition in the target in the case of source activation. In this study, we examine the top-down and interhemispheric modulatory connections in a visual network, using a perceptual task that involves interhemispheric spatial integration [10, 11]. Spatial integration refers to the brain processes that implement a global representation of spatially extended objects by assembling local information across the visual field. In contrast to the majority of visual functions that achieve adult levels within a few months from birth, spatial integration develops at least until midadolescence [12-15]. Migraine interferes with the development of spatial integration such that, compared to agematched controls, children with migraine show reduced improvement in the performance of contour integration task between 6 and 14 years [16].
Our task contrasts colinear and orthogonal bilateral gratings, of which only the first generates a Gestalt such that the stimulus parts are fused between hemispheres. In agreement with predictive coding theory, a more coherent (colinear) stimulus would cause activations in higher-order and deactivations in lower-order visual areas [17]. Indeed this phenomenon has been observed in adults performing visual integration tasks $[10,11,18-20]$. As recently shown with DCM, such dynamics in adults depends on top-down and lateral inhibition [21, 22] and is weakened with age and all the more so in Alzheimer's disease [22]. Yet nothing is known about the development of top-down effects within the human visual cortices.

We hypothesized that the functional circuits providing top-down effects continue to advance during the second decade of human life. In addition to characterizing their developmental changes from a network perspective, we aimed at linking them to structural brain maturation as manifested by interhemispheric callosal connectivity. Here, we report the DCM-based evidence of this process in healthy children, a necessary step for the evaluation of the state of long-developing networks in children with neurological disorders including migraine. Some of these results have been mentioned elsewhere in relation to the corpus callosum development [23].

\section{Materials and methods}

\section{Subjects}

The data from 13 children (4 girls, 9 boys, mean age 11.0 years, range 7-13 years) with normal vision and without known neurological or psychiatric illness have been analyzed in the study. Previously we have used the data from this population to track the functional and morphological maturation of interhemispheric connectivity with a combined MRI technique [15]. Fourteen normal adults ( 6 women and 8 men; mean age 29.8 years, range of 21-42 years) without known neurological or psychiatric conditions and with normal or corrected-to-normal vision also participated in the study. The study followed the protocol approved by the Lausanne University Ethics Committee and written informed consent was obtained from children and their parents as well as from the adult participants. All the instrumental procedures conformed to the Declaration of Helsinki (1964) of the World Medical Association concerning experimentation on humans.

Stimuli

Subjects viewed bilateral sinusoidal black-and-white gratings with a spatial frequency of $0.5 \mathrm{~Hz}$, a contrast of $70 \%$, 
a size of a lateral patch of $11^{\circ} \times 19^{\circ}$, and drifting with a temporal frequency of $2 \mathrm{~Hz}$. The stimuli either obeyed Gestalt grouping rules [iso-oriented colinear gratings (CG)] or violated them [non-colinear orthogonally oriented (NG)]. The stimulus conditions were alternated with background (uniform gray screen of the same space-averaged luminance as the stimuli) in a balanced pseudo-randomized order, five (for children) or six times (for adults), $15 \mathrm{~s}$ each (for a detailed description of the stimuli and eye movement control see $[10,11])$.

\section{fMRI data acquisition and pre-processing}

For children, the protocol was performed on a 1.5 Tesla Siemens Magnetom Vision. Functional MRI images were acquired with an EPI gradient echo sequence (FA 90 ${ }^{\circ}$, TE $66 \mathrm{~ms}$, pixel size $3.75 \times 3.75 \mathrm{~mm}^{2}$, acquisition time $1.7 \mathrm{~s}$, 22 slices, $5 \mathrm{~mm}$ thick) with a TR $3 \mathrm{~s}$.

The adults were imaged on a $3 \mathrm{~T}$ Siemens Trio scanner with the same type of sequence (FA $90^{\circ}$, TE $30 \mathrm{~ms}$, pixel size $3 \times 3 \mathrm{~mm}^{2}$, acquisition time $1.7 \mathrm{~s}, 32$ slices, $3 \mathrm{~mm}$ thick, TR $2 \mathrm{~s}$ ). As the structural basis for brain segmentation and surface reconstruction, we acquired high-resolution T1-weighted 3D gradient echo sequence (MPRAGE), 160 slices $(1 \times 1 \times 1 \mathrm{~mm}$ voxel size $)$. In both experiments, we prevented head movements by cushioning the participant's head in a padded coil.

Both fMRI datasets were pre-processed and analyzed using Statistical Parametric Mapping (SPM8, Wellcome Department of Cognitive Neurology, London, UK), first, for standard single-subject and group statistics, then for DCM-specific design. Functional images were realigned to the first scan using a six-parameter rigid-body transformation and co-registered to the respective anatomical acquisition. To minimize inter-subject anatomical differences and increase the signal-to-noise ratio, each subject's images were normalized to the Montreal Neurological Institute template (MNI) using a 12-parameter affine transformation [24], re-sampled to $2 \times 2 \times 2 \mathrm{~mm}^{3}$, and smoothed with an isotropic Gaussian kernel $(\mathrm{FWHM}=6 \mathrm{~mm})$.

It should be mentioned that moderately stronger and more extensive activations that can be recorded with the $3 \mathrm{~T}$ compared to $1.5 \mathrm{~T}$ scanner in a visual perceptual task [25] do not compromise the validity of comparative DCM analysis, since the method models the temporal dynamics of BOLD signals rather than their intensity. Furthermore, the local character of the modeled network situated within the visual cortex suggests the homogeneity of magnetic field across the regions of interest (ROIs). Finally, our ROIs (for details see "ROIs for DCM") are similarly located in the adult data from $1.5 \mathrm{~T}[10,11]$ and $3 \mathrm{~T}$ (here), and, therefore, represent the same network.
Single-participant analysis was performed by adjusting the general linear model to our block design experiment. The signal drift across acquisitions was removed with a high-pass filter. Statistical parametric maps of the contrasts of interest were computed for each subject and were used as input values for the group statistics based on random field theory. In particular, the inferential statistics included between-condition $t$ tests. To identify the nodes for DCM analysis (hereafter referred to as ROIs), we considered centers of clusters surviving a statistical threshold for peak height at $P<0.05$ [family wise error (FWE) corrected] and satisfying an extent threshold of $k>30$ contiguous voxels in the group SPMs.

\section{Dynamic causal modeling}

DCM serves to assess condition-dependent interactions within the distributed brain network based on an fMRI time series. To this end, DCM considers the brain as a dynamic system, in which external inputs cause changes in neuronal activity, which, in turn, cause changes in the distributed fMRI signals [9]. Mathematically, DCM for fMRI includes a bilinear model of neural dynamics and an extended balloon hemodynamic model of the transformation of neural activity into a measured BOLD response [9, 26]. A fixed set of ROIs, possible connections between them, and driving inputs are prerequisites for determining effective connectivity, which is defined by the influences that one node in a model exerts over another. Effective connections are divided into the intrinsic connections, i.e., the influences between ROIs in the absence of a modulating context, and the modulatory connections, i.e., the changes due to the experimental context. Effective connections can be positive, i.e., excitation in a source region induces increase in activity in the target, and negative, i.e., functional activation in the source causes target inhibition. Since the theoretical and practical issues of DCM were recently reviewed, we refer interested readers to these sources for details of the method and underlying theory [27-29].

\section{Roles for DCM}

The results of standard GLM analysis of SPMs reported earlier [10, 15] served as the basis for ROI selection. Both children and adults showed extensive activations in the striate and extrastriate areas under $\mathrm{CG}$ and $\mathrm{NG}$ conditions. In the primary visual cortex, we have chosen ROIs centered on the local group maxima from the conjunction maps of $\mathrm{CG}>$ background and $\mathrm{NG}>$ background contrasts. In the extrastriate areas, we defined ROIs from the $\mathrm{CG}>\mathrm{NG}$ contrast, which revealed bilateral activations in the lingual gyrus (V3v, Fig. 1) in both groups. At the group level, the local maxima within these activated clusters served as ROI 


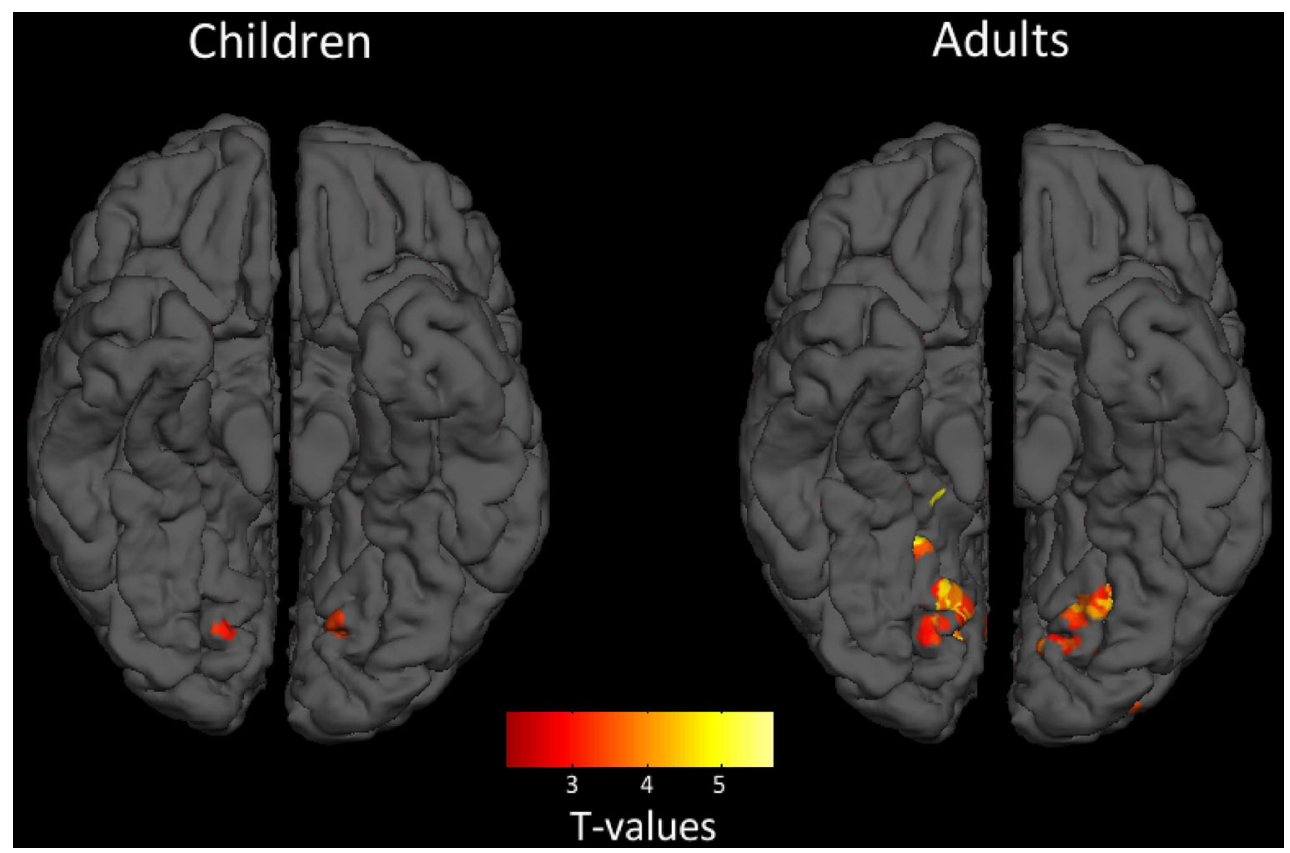

Fig. 1 Interhemispheric integration effects as revealed by fMRI activation. The 3D representation of statistical maps for the contrast between colinear and non-colinear gratings in the groups of children and adults $(P<0.001$, extent threshold $k=30$ voxels $)$ is rendered on the MNI canonical brain. The bottom view of a pial surface is presented. In adults, the MNI coordinates $(x, y, z)$ of the center of lefthemisphere cluster (178 voxels) are $-12,-70,-10$ and those of

Table 1 Anatomical locations of regions activated by gratings

\begin{tabular}{|c|c|c|c|c|}
\hline \multirow[t]{3}{*}{ Group } & \multicolumn{2}{|c|}{$\mathrm{CG}>$ background } & \multicolumn{2}{|l|}{$\mathrm{CG}>\mathrm{NG}$} \\
\hline & \multicolumn{4}{|l|}{ Hemisphere } \\
\hline & Left & Right & Left & Right \\
\hline Adults & $\begin{array}{l}59 \% \mathrm{~V} 1, \\
41 \% \mathrm{~V} 2\end{array}$ & $\begin{array}{r}66 \% \mathrm{~V} 1 \\
34 \% \mathrm{~V} 2\end{array}$ & $\begin{array}{r}58 \% \mathrm{~V} 3 \mathrm{v} \\
42 \% \mathrm{~V} 4\end{array}$ & $\begin{array}{r}65 \% \mathrm{~V} 3 \mathrm{v} \\
35 \% \mathrm{~V} 4\end{array}$ \\
\hline Children & $\begin{array}{l}62 \% \mathrm{~V} 1, \\
38 \% \mathrm{~V} 2\end{array}$ & $\begin{array}{l}69 \% \mathrm{~V} 1, \\
31 \% \mathrm{~V} 2\end{array}$ & $\begin{array}{r}51 \% \mathrm{~V} 3 \mathrm{v} \\
49 \% \mathrm{~V} 4\end{array}$ & $\begin{array}{r}70 \% \mathrm{~V} 3 \mathrm{v} \\
30 \% \mathrm{~V} 4\end{array}$ \\
\hline
\end{tabular}

Functional locations of the centers of activated clusters are given according to a probabilistic atlas [61]. The percentage represents each center's mean probability of belonging to the mentioned visual areas across subjects

centers. In individual subjects, the location of an ROI center was determined as a local maximum closest to the respective group location.

For each subject and ROI, the first principal component of the original time series of all voxels within a 4-mm radius sphere was extracted. In the primary visual cortex, ROIs were located at the V1/V2 border (Table 1). The ROIs in the extrastriate cortex occupied territory in V3v/V4. Hereafter, for the sake of simplicity, these two pairs of ROIs are referred to as $\mathrm{V} 1_{\mathrm{L}}, \mathrm{V} 1_{\mathrm{R}}, \mathrm{V} 3_{\mathrm{L}}$, and $\mathrm{V} 3_{\mathrm{R}}$, where the "L" and " $R$ " designate the left and right hemispheres, respectively. right-hemisphere cluster (133 voxels) are $16,-76,-14$. In children, the coordinates are $-18,-78,-14$ (left hemisphere, 88 voxels) and $10,-76,-6$ (right hemisphere, 95 voxels). Hot scale shows $T$ values. In both groups, BOLD response is located within the lingual gyri, but the clusters are larger and more significant in the adult group. The centers of the clusters $(P<0.05$, FWE corrected $)$ define the V3 locations for DCM analysis

\section{Dynamic causal model}

For the sake of compatibility between children and adults, we applied a single model to both groups (Fig. 2). This is a fully connected model of intrinsic connections, which has also been a winning one for the group of aged healthy adults under the same perceptual task [22]. In this model, the stimuli $>$ background contrast serves as a driving input. The signals induced by the bilateral stimuli travel via crossed visual pathways and directly affect the left and right primary visual cortices. The induced activity then spreads through the system according to the intra- and interhemispheric reciprocal connections. On the assumption that each intrinsic connection can be modulated by the task, we reproduced the architecture of intrinsic connections for modulatory connections (Fig. 3). We modeled the CG $>$ stimuli contrast, since in our experiment the interhemispheric integration is a source of modulatory effects.

\section{Group statistics for DCM}

To test for differences in the coupling parameters between developing and mature brain networks, we applied mixed between-within analysis of variance (ANOVA). The developmental affects were analyzed with a between- 


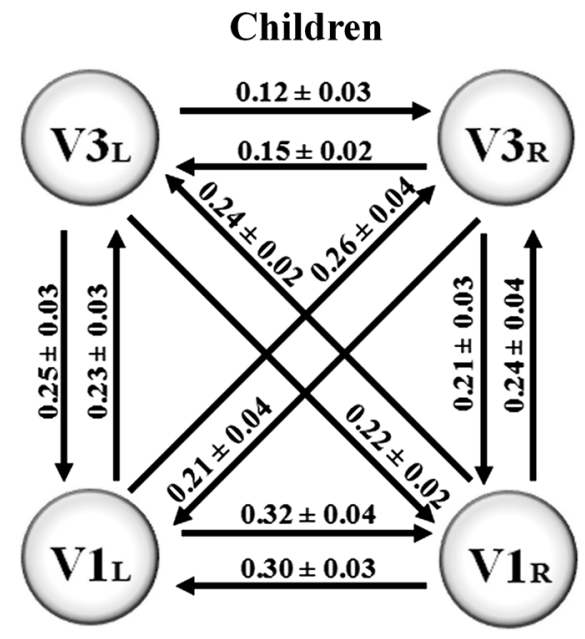

Fig. 2 Intrinsic connections in children and adults. The schematized representation of the modeled networks includes gray-filled circles, which denote ROIs for DCM analysis, and arrows, which stand for intrinsic connections. The ROIs are located in the $\mathrm{V} 1_{\mathrm{L}}, \mathrm{V} 1_{\mathrm{R}}, \mathrm{V} 3_{\mathrm{R}}$, and $\mathrm{V} 3_{\mathrm{L}}$. The average strength of a connection in Hertz is shown together

subject age factor (children vs. adults). According to our hypothesis, developmental effects might differ depending on the topography of connections, which, in terms of ANOVA, can be shown with an interaction of the topography of connections with the age factor. The planned between-group comparisons of connection parameters were performed by means of a two-sample two-tailed $t$ test. For descriptive statistics of each parameter, we used a onesample two-tailed $t$ test. Significant effects are reported at $P<0.05$ corrected for multiple comparisons with the Bonferroni method.

The links between the coupling parameters of the DC model and morphometric measurements of the corpus callosum (CC) were assessed by the Pearson correlation coefficient $\rho$ with corresponding $P$ value. All the statistical tests were implemented with SPSS 17.0 for Windows.

\section{Results}

Intrinsic connections and driving inputs do not change with age

Group-averaged estimates of the strength of intrinsic connections are shown in Fig. 2. All the parameters significantly differ from zero at $P<0.05$ (two-tailed $t$ test, Bonferroni corrected). To explore the age-related changes in vertical effective connections between striate (V1) and extrastriate (V3) areas, including both intra- and interhemispheric connections, we applied a three-way betweenwithin ANOVA with factors of age (children vs. adults), direction (top-down vs. bottom-up), and topography (4

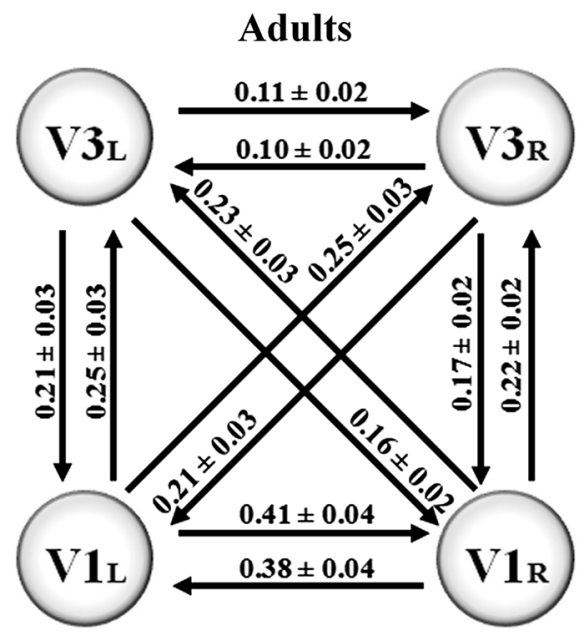

with a standard error next to the respective connection. For the sake of simplicity, the driving inputs provided by the right visual field gratings to $V 1_{L}$ and by the left visual field gratings to $V 1_{R}$ are not shown

levels: $\quad \mathrm{V} 1_{\mathrm{L}}-\mathrm{V} 3_{\mathrm{L}}, \quad \mathrm{V} 1_{\mathrm{L}}-\mathrm{V} 3_{\mathrm{R}}, \quad \mathrm{V} 1_{\mathrm{R}}-\mathrm{V} 3_{\mathrm{L}}, \quad$ and $\mathrm{V} 1_{\mathrm{R}}-\mathrm{V} 3_{\mathrm{R}}$ ). Neither the main effect of age, nor its interactions with the other two factors proved significant. We also failed to find age-related changes in horizontal interhemispheric connections at the striate and extrastriate levels, within the framework of a three-way betweenwithin ANOVA with factors of age (children vs. adults), hierarchy (V1 vs. V3), and direction [V1(3) $)_{\mathrm{L}} \rightarrow \mathrm{V} 1(3)_{\mathrm{R}} \mathrm{vs}$. $\left.\mathrm{V} 1(3)_{\mathrm{L}} \leftarrow \mathrm{V} 1(3)_{\mathrm{R}}\right]$.

Similarly, we found no differences in driving inputs between the groups or hemispheres as shown with a twoway between-within ANOVA with factors of age (children vs. adults) and hemisphere (left vs. right).

Modulatory connections strengthen with age

All the modulatory connections turned out to be inhibitory (Fig. 3). Six out of 12 connections in children and all but one in adults significantly differ from zero at $P<0.05$ (two-tailed $t$ test, Bonferroni corrected). Note that significant connections in children are mostly bottom-up. The vertical modulatory connections were analyzed with a twoway between-within ANOVA, which includes the age and topography factors described for intrinsic connections. The main effect of age was significant at $P=0.037$ ( $F=4.88$, $\left.d f=1, \eta^{2}=0.163\right)$. As Fig. $3 \mathrm{~b}$ shows, the strength of inhibitory top-down connections builds up with age. A three-way between-within ANOVA with the age, hierarchy, and direction factors (described for horizontal intrinsic connections) revealed the main effect of age $(P<0.001$, $F=18.289, d f=1, \eta^{2}=0.422$ ) for horizontal modulatory connections, which appeared to be stronger in adults. 
A
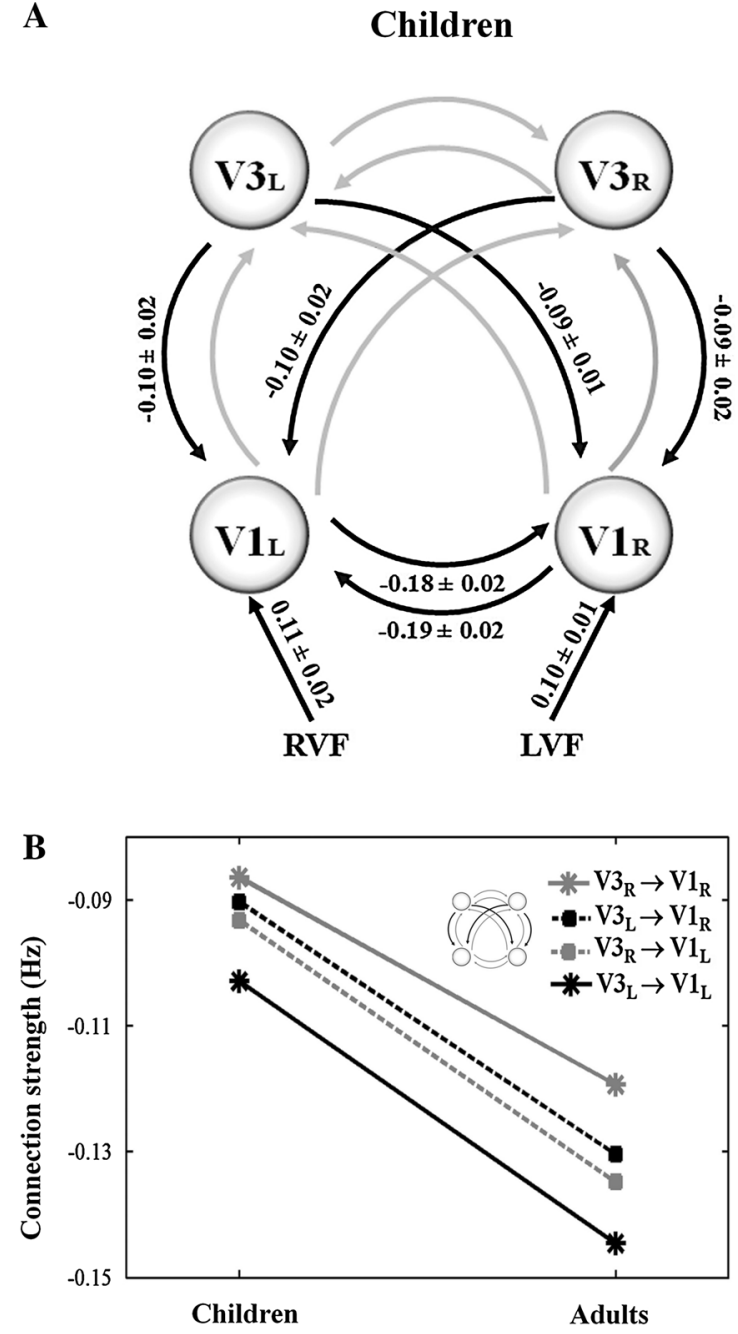

Fig. 3 Modulatory connections for children and adult groups. a The average estimates of modulatory parameters in Hertz with standard errors are shown alongside the respective connections. Other designations are as in Fig. 2. b The main effect of age on top-down

This effect interacted with a hierarchy factor at $P=0.007$ $(F=8.589, \quad d f=1, \quad$ Greenhouse-Geisser corrected; $\eta^{2}=0.256$ ). The planned between-group comparisons showed that only striate connections are significantly stronger in adults than in children $(P=0.001$, $\mathrm{V} 1_{\mathrm{L}} \rightarrow \mathrm{V} 1_{\mathrm{R}} ; P=0.013, \mathrm{~V} 1_{\mathrm{L}} \leftarrow \mathrm{V} 1_{\mathrm{R}}$, Fig. 3c).

Effective coupling is associated with brain maturation

To visualize the developmental trajectory for interhemispheric effective connectivity and to link it to structural brain maturation, we applied a polynomial regression analysis to the effective connections between primary visual areas that were significantly stronger in adults than in children. To this end, age and the CC area assessed on the basis of a midsagittal slice served as estimators of general development and of structural maturation,
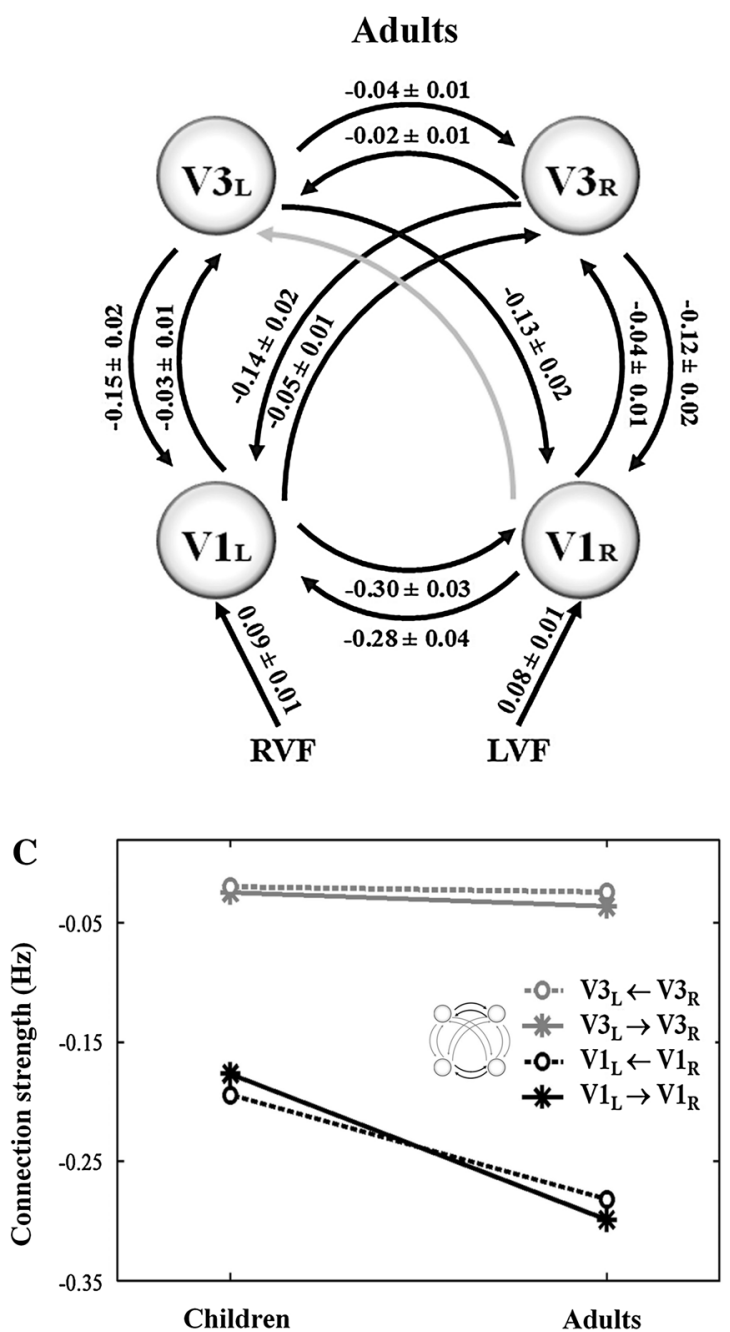

modulatory connections. Connections from left and right hemispheres are in black and gray, respectively. c The main effects of the age factor and the interaction of age $\times$ hierarchy on the striate (in black) and extrastriate (in gray) interhemispheric connections

respectively. The average strength of the $\mathrm{V} 1_{\mathrm{L}} \rightarrow \mathrm{V} 1_{\mathrm{R}}$ and $\mathrm{V} 1_{\mathrm{L}} \leftarrow \mathrm{V} 1_{\mathrm{R}}$ modulatory connections was used as a dependent variable. We found that both linear and quadratic regression models are significant for age $(P=0.002$; Fig. 4a) and for the CC area $(P=0.004$ for the linear and $P=0.001$ for the quadratic model; Fig. 4 b). The quadratic models showed better fit to the data (41.1 and $44.9 \%$ of variance for age and $\mathrm{CC}$ area, respectively) than the linear models (34.3 and $30.3 \%$ ).

Although the CC area correlates with age $(\rho=-0.62$, $P=0.001)$, the latter is a more general index, accumulating all the developmental changes. To assess the contribution of the individual variations of the $\mathrm{CC}$ area, while controlling for age, we applied hierarchical multiple regression analysis. Preliminary analysis ensured no violations of the assumptions of normality, linearity, and multi-colinearity (tolerance $=0.62$ ). In the hierarchical 
A

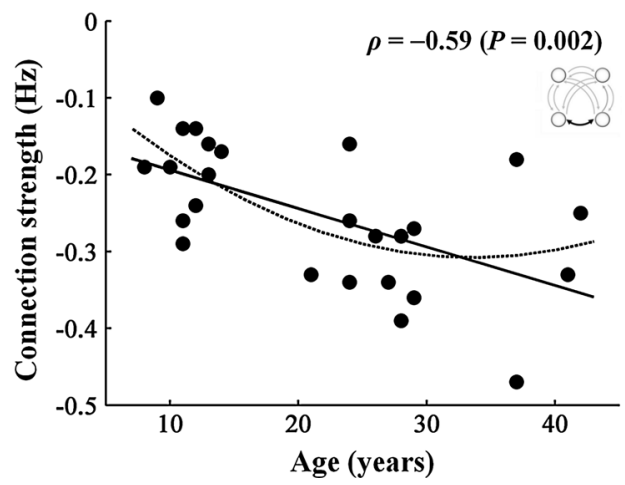

Fig. 4 Effective connectivity and structural brain maturation. The plots show scatter diagrams and developmental trajectories fitted with the first- and second-level polynoms (solid and dashed line, respectively) for age (a) and the CC area (b). Pearson regression coefficient $\rho$ with corresponding $P$ value represents a measure of bivariate

regression model with the two variables, which turned out to be significant at $P=0.003$ and explained $40 \%$ of the variance in connection strength, the $\mathrm{CC}$ area predicted only $5.7 \%$ in addition to what age predicted. Therefore, being controlled for the effect of age, the $\mathrm{CC}$ area failed to explain a significant amount of variance.

\section{Discussion}

Development of spatial integration and changes in top-down effective connections

In children, the key mechanisms of spatio-temporal integration are functional beginning in infancy. In particular, newborns looking at point-light animations prefer biological motion to non-biological motion and, among the biomotion animations, distinguish between upright and inverted displays [30]. Beginning at 2 months of age, infants combine the coherently moving components across large areas of space and treat them as connected wholes [31]. By 3-4 months of age, they are able to perceive the global structure of various arrays [32]. Further development of visual spatial integration continues through middle childhood and, probably, adolescence. Sensitivity to the global structure of Glass patterns with a different percentage of noise approximates that of adults at 9 years of age [33]. The capacity to integrate spatially distant line segments based on their colinearity improves until 10 years of age [34]. Spatial integration tested in contour-detection tasks improves until about 14 years of age [12]. A similar trajectory was shown for sensitivity to biological and global motion [35]. Furthermore, the processing of global shape in paradigms including compound displays still differs in adolescence from that in adults $[36,37]$.

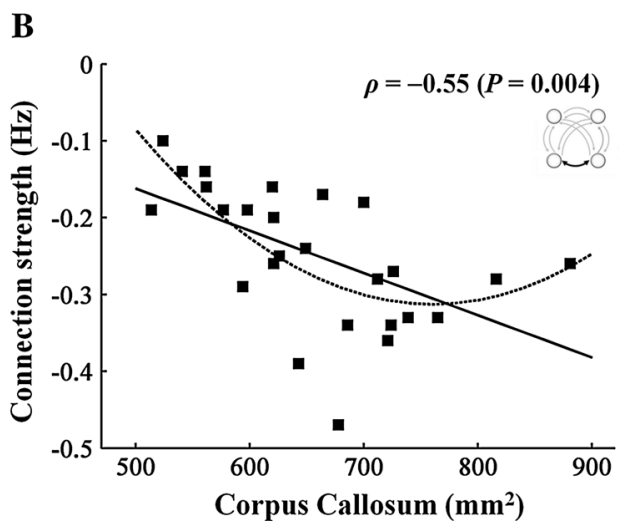

correlation. The $\mathrm{CC}$ area was assessed from a midsagittal slice. The connection strength refers to the average value of reciprocal modulatory connections between the primary visual (black line on the connection scheme)

These behavioral changes are paralleled by the development of functional connectivity in neural networks as manifested by EEG synchronization [38-41]. To show the transformations of neural networks underlying the protracted maturation of integrative mechanisms, here we have applied the DCM method to an fMRI time series under a passive perceptual task, which contrasted bilateral colinear gratings (Gestalt) to bilateral orthogonal gratings (no Gestalt), of which only the first stimulus can be interhemispherically integrated.

Our analysis is implemented in the framework of predictive coding theory, which provides an elegant model of the hierarchical optimization mechanism. It is based on feedback connections that carry predictions of lower-level neural activity and feed-forward connections that carry residual errors between predictions and actual lower-level responses $[8,17]$. Indeed, a DCM analysis of fMRI activations induced by coherent or meaningful stimuli compared to random ones has shown inhibitory modulatory effects from the higher-order to the lower-order visual areas in adult subjects [21].

Here, we have shown that intrinsic effective connections within the visual areas do not differ between children older than 7 years and young adults, suggesting that basic visual networks integrated via long-distance reciprocal excitatory pathways are established at this age. The intrinsic connections were modulated by the coherent CG stimulus both in children and in adults. All the descending and lateral modulatory connections turned out to be inhibitory. Therefore, here we have shown for the first time that inhibitory backward effective coupling recently reported in adults [21, 22] works in children as well.

This result agrees with a large body of literature reporting the higher-order effects on V1 via feedback connections originating within the visual system and/or via 
long-range local horizontal connections [42, 43]. Although low-level integration has been predominantly studied intrahemispherically as a contextual modulation in the neighborhood of the classical receptive field [44, 45], in adults it likely operates at a long range as well, e.g., across the cerebral hemispheres. In this regard, Ban and colleagues have demonstrated that interhemispheric integration can result in changes of V1 activation [20]. They have found a significantly lower BOLD response to two arcs located symmetrically in the lower visual field quadrants (i.e., to a pattern following the Gestalt principle of good continuation) compared to their asymmetrical (diagonal) location. In the absence of direct interhemispheric V1 connections between the low and high visual quadrants, this modulation of V1 activation is likely due to the topdown influences from the extrastriate areas.

The finding that the top-down modulatory effects in adolescents differ from those in adults suggests their slow formation in human ontogenesis. The available data on the structural maturation of feedback connections, which might be a part of the neural network that enables colinearity detection in humans [12], are limited to those between V2 and V1 [1, 2]. According to this postmortem anatomical study, the upper layers of $\mathrm{V} 1$, which receive the feedback connections, seem to be immature even at 5 years of age. In contrast, we found no evidence supporting the immaturity of effective connections downstream of $\mathrm{V} 1$, which have also been proposed to underlie the slow progression of perceptual integration in human ontogenesis [35].

The abnormal development of top-down connections in children can explain a well-known interictal phenomenon of reduced habituation to repetitive visual and auditory stimuli in migraine patients [46-48]. Sensory habituation, characteristic for healthy adults and children, refers to progressive reduction in the cortical response to repetitive stimuli. It is manifested as a gradual decrease in the amplitude of evoked potentials. Habituation can be considered as a mechanism protecting against the accumulation of potentially toxic metabolites in the brain. According to the predictive coding hypothesis, the repetition reduces cortical response due to increasing predictability of successive stimuli emerging as a direct result of predictive processing in the brain [49]. Since this effect is sustained through inhibitory feedback connections, their formation in children, which suffer from migraine, should be thoroughly explored in future studies.

\section{Interhemispheric modulation in V1}

In addition to feedback connections strengthening with age, we found increasing interhemispheric inhibitory effects in the primary visual cortex. More precisely, according to the probabilistic cytoarchitectonic atlas, our ROIs designated as V1 included a portion of V2 as well (Table 1). The effects relevant to these DCM results have been recently shown in animal and human electrophysiological studies. Regarding animals, interhemispheric modulatory effects near the V1/V2 border have been observed in a ferret model [50]. As in our experiment, colinear or orthogonal gratings were presented to the two visual hemifields. They induced desynchronization of the local field potential, which could be modulated by reversible cooling of the opposite V1 under the colinear condition.

In adult humans, interhemispheric inhibition in the primary visual cortex has been shown with unilateral transcranial magnetic stimulation of $\mathrm{V} 1$, which increased (by means of disinhibition) the amplitude of EEG potentials, evoked by gratings, in the contralateral hemisphere [51]. Another example of transcallosal inhibition in the human primary visual cortex has been demonstrated in binocular rivalry. During perceptual transitions, a dominant stimulus can spread across the visual field in a wave-like manner. These traveling waves of rivalry dominance are delayed when passing from one visual hemifield to another. In a diffusion tensor imaging study, the diffusion properties of callosal fibers connecting the left and right V1 reliably predicted this across-hemifield delay [52]. Since perceptual alternation during binocular rivalry is likely to result from inhibitory interactions between neural representations of the different percepts [53], this finding links the CC to interhemispheric inhibition.

Hypothetically, the insufficiency of interhemispheric inhibition between the primary visual areas could be among the factors behind the reduced habituation (the increased amplitude of visual evoked potential) in patients with migraine. However, until now only the late components of visual evoked potentials, generated in the extrastriate areas, are shown to be different (e.g., [54]).

From an ontogenetic perspective, immature transcallosal modulation between the primary visual areas in the absence of age-dependent interhemispheric effects in the extrastriate areas challenges the conventional view that posits the prior maturation of the early visual cortex as a precondition for the later development of higher-order ventral stream regions [55]. Yet our finding is supported by the association between the indices of effective connectivity and brain maturation. The interhemispheric interaction in the ventral visual stream is implemented via fibers that form the splenium of the CC [56, 57]. Poorly myelinated fibers of small diameter with a protracted course of myelination are abundant in this region [58]. The CC area depends on the number of axons, their size, and the thickness of the myelin sheaths [15, 59], providing a reasonable cumulative index of interhemispheric connectivity. As we found, its agedependent changes explain about $40 \%$ of the variance in 
interhemispheric effective inhibition between the primary visual areas.

Since interhemispheric effective inhibition is presumably implemented via polysynaptic pathways with longdistance excitatory and local inhibitory components, this correlation reflects only the development of the long-distance part. As far as local inhibitory mechanisms in the primary visual cortex are concerned, they were recently analyzed postmortem [60]. Consistently with our results, this study revealed the protracted development of GABAergic mechanisms, which continues well into the second and third decades of life.

In sum, our results suggest that feedback connections from the extrastriate to the primary visual areas together with the interhemispheric connections between primary visual areas strongly contribute to spatio-temporal feature integration in the mature brain. Both feedback and horizontal effective connectivity have a long developmental trajectory extending into the adolescence. This wide developmental window suggests a protracted period of the vulnerability to neuropathological disorders including migraine. For better understanding of the mechanism inducing migraine, the DCM can be used. The method seems to be especially efficient for the analysis of brain networks providing habituation.

Acknowledgments This work was supported by the Centre d'Imagerie BioMédicale (CIBM) of the University of Lausanne (UNIL), the Swiss Federal Institute of Technology Lausanne (EPFL), the University of Geneva (UniGe), the Centre Hospitalier Universitaire Vaudois (CHUV), the Hôpitaux Universitaires de Genève (HUG) and the Leenaards and the Jeantet Foundations.

Conflict of interest All the authors certify that there is no actual or potential conflict of interest in relation to this article.

\section{References}

1. Burkhalter A (1993) Development of forward and feedback connections between areas V1 and V2 of human visual cortex. Cereb Cortex 3:476-487

2. Burkhalter A, Bernardo KL, Charles V (1993) Development of local circuits in human visual cortex. J Neurosci 13:1916-1931

3. Stevens MC, Kiehl KA, Pearlson GD, Calhoun VD (2007) Functional neural networks underlying response inhibition in adolescents and adults. Behav Brain Res 181:12-22

4. Hwang K, Velanova K, Luna B (2010) Strengthening of topdown frontal cognitive control networks underlying the development of inhibitory control: a functional magnetic resonance imaging effective connectivity study. J Neurosci 30:15535-15545

5. Filippi M, Rocca MA (2008) Headache and migraine. Neurol Sci 29(Suppl 3):336-338

6. Chiapparini L, Ferraro S, Grazzi L, Bussone G (2010) Neuroimaging in chronic migraine. Neurol Sci 31(Suppl 1):S19-S22

7. Abu-Arefeh I, Russell G (1994) Prevalence of headache and migraine in school children. BMJ 309:765-769
8. Rao RP, Ballard DH (1999) Predictive coding in the visual cortex: a functional interpretation of some extra-classical receptivefield effects. Nat Neurosci 2:79-87

9. Friston KJ, Harrison L, Penny W (2003) Dynamic causal modelling. Neuroimage 19:1273-1302

10. Knyazeva MG, Fornari E, Meuli R, Innocenti G, Maeder P (2006) Imaging of a synchronous neuronal assembly in the human visual brain. Neuroimage 29:593-604

11. Knyazeva MG, Fornari E, Meuli R, Maeder P (2006) Interhemispheric integration at different spatial scales: the evidence from EEG coherence and FMRI. J Neurophysiol 96:259-275

12. Kovacs I, Kozma P, Feher A, Benedek G (1999) Late maturation of visual spatial integration in humans. Proc Natl Acad Sci USA 96:12204-12209

13. Atkinson $\mathbf{J}$ (2000) The developing visual brain. Oxford University Press, Oxford, p 211

14. Norcia A (2003) Development of spatial selectivity and response timing in humans. In: Werner JS, Chalupa LM (eds) The visual neurosciences. MIT Press, Cambridge, pp 1603-1615

15. Fornari E, Knyazeva MG, Meuli R, Maeder P (2007) Myelination shapes functional activity in the developing brain. Neuroimage 38:511-518

16. Braunitzer G, Rokszin A, Kobor J, Nagy A, Sztriha L, Benedek G (2011) Development of visual contour integration in children with migraine without aura. Cephalalgia 31:1048-1056

17. Friston K, Kiebel S (2009) Predictive coding under the freeenergy principle. Philos Trans R Soc Lond B Biol Sci 364:1211-1221

18. Murray SO, Schrater P, Kersten D (2004) Perceptual grouping and the interactions between visual cortical areas. Neural Netw 17:695-705

19. Braddick OJ, O'Brien JM, Wattam-Bell J, Atkinson J, Hartley T, Turner R (2001) Brain areas sensitive to coherent visual motion. Perception 30:61-72

20. Ban H, Yamamoto H, Fukunaga M, Nakagoshi A, Umeda M, Tanaka C, Ejima Y (2006) Toward a common circle: interhemispheric contextual modulation in human early visual areas. J Neurosci 26:8804-8809

21. Cardin V, Friston KJ, Zeki S (2011) Top-down modulations in the visual form pathway revealed with dynamic causal modeling. Cereb Cortex 21:550-562

22. Rytsar R, Fornari E, Frackowiak RS, Ghika JA, Knyazeva MG (2011) Inhibition in early Alzheimer's disease: an fMRI-based study of effective connectivity. Neuroimage 57:1131-1139

23. Knyazeva MG (2013) Splenium of corpus callosum: patterns of interhemispheric interaction in children and adults. Neural Plast 2013:639430

24. Ashburner J, Friston KJ (2000) Voxel-based morphometry-the methods. Neuroimage 11:805-821

25. Krasnow B, Tamm L, Greicius MD, Yang TT, Glover GH, Reiss AL, Menon V (2003) Comparison of fMRI activation at 3 and 1.5 $\mathrm{T}$ during perceptual, cognitive, and affective processing. Neuroimage 18:813-826

26. Buxton RB, Wong EC, Frank LR (1998) Dynamics of blood flow and oxygenation changes during brain activation: the balloon model. Magn Reson Med 39:855-864

27. Seghier ML, Zeidman P, Neufeld NH, Leff AP, Price C (2010) Identifying abnormal connectivity in patients using dynamic causal modeling of fMRI responses. Front Syst Neurosci 4:142

28. Stephan KE, Friston KJ (2010) Analyzing effective connectivity with functional magnetic resonance imaging. Wiley Interdiscip Rev Cogn Sci 1:446-459

29. Friston KJ, Li B, Daunizeau J, Stephan KE (2011) Network discovery with DCM. Neuroimage 56:1202-1221 
30. Simion F, Regolin L, Bulf H (2008) A predisposition for biological motion in the newborn baby. Proc Natl Acad Sci USA 105:809-813

31. Spelke ES (1990) Principles of object perception. Cogn Sci 14:29-56

32. Humphrey GK, Humphrey DE, Muir DW, Dodwell PC (1986) Pattern perception in infants: effects of structure and transformation. J Exp Child Psychol 41:128-148

33. Lewis TL, Ellemberg D, Maurer D, Dirks M, Wilkinson F, Wilson HR (2004) A window on the normal development of sensitivity to global form in Glass patterns. Perception 33:409-418

34. Hadad BS, Kimchi R (2006) Developmental trends in utilizing perceptual closure for grouping of shape: effects of spatial proximity and collinearity. Percept Psychophys 68:1264-1273

35. Hadad BS, Maurer D, Lewis TL (2011) Long trajectory for the development of sensitivity to global and biological motion. Dev Sci 14:1330-1339

36. Mondloch CJ, Geldart S, Maurer D, de Schonen S (2003) Developmental changes in the processing of hierarchical shapes continue into adolescence. J Exp Child Psychol 84:20-40

37. Scherf KS, Behrmann M, Kimchi R, Luna B (2009) Emergence of global shape processing continues through adolescence. Child Dev 80:162-177

38. Farber DA, Knyazeva MG (1991) Electrophysiological correlates of interhemispheric interaction in ontogenesis. In: Ramaekers G, Njiokiktjien C (eds) Pediatric behavioural neurology. Suyi Publications, Amsterdam, pp 86-99

39. Innocenti GM, Kiper DC, Knyazeva MG, Deonna TW (1999) On nature and limits of cortical developmental plasticity after an early lesion, in a child. Restor Neurol Neurosci 15:219-227

40. Knyazeva MG, Innocenti GM (2001) EEG coherence studies in the normal brain and after early-onset cortical pathologies. Brain Res Brain Res Rev 36:119-128

41. Knyazeva MG, Maeder P, Kiper DC, Deonna T, Innocenti GM (2002) Vision after early-onset lesions of the occipital cortex: II. Physiological studies. Neural Plast 9:27-40

42. Bullier J, Hupe JM, James AC, Girard P (2001) The role of feedback connections in shaping the responses of visual cortical neurons. Prog Brain Res 134:193-204

43. Gilbert CD, Sigman M (2007) Brain states: top-down influences in sensory processing. Neuron 54:677-696

44. Altmann CF, Bulthoff HH, Kourtzi Z (2003) Perceptual organization of local elements into global shapes in the human visual cortex. Curr Biol 13:342-349

45. Kourtzi Z, Tolias AS, Altmann CF, Augath M, Logothetis NK (2003) Integration of local features into global shapes: monkey and human FMRI studies. Neuron 37:333-346
46. Coppola G, Pierelli F, Schoenen J (2009) Habituation and migraine. Neurobiol Learn Mem 92:249-259

47. Schoenen J (2006) Neurophysiological features of the migrainous brain. Neurol Sci 27(Suppl 2):S77-S81

48. Pro S, Tarantino S, Capuano A, Vigevano F, Valeriani M (2014) Primary headache pathophysiology in children: the contribution of clinical neurophysiology. Clin Neurophysiol 125:6-12

49. Clark A (2013) Whatever next? Predictive brains, situated agents, and the future of cognitive science. Behav Brain Sci 36:181-204

50. Carmeli C, Lopez-Aguado L, Schmidt KE, De Feo O, Innocenti GM (2007) A novel interhemispheric interaction: modulation of neuronal cooperativity in the visual areas. PLoS One 2:e1287

51. Bocci T, Caleo M, Giorli E, Barloscio D, Maffei L, Rossi S, Sartucci F (2011) Transcallosal inhibition dampens neural responses to high contrast stimuli in human visual cortex. Neuroscience 187:43-51

52. Genc E, Bergmann J, Tong F, Blake R, Singer W, Kohler A (2011) Callosal connections of primary visual cortex predict the spatial spreading of binocular rivalry across the visual hemifields. Front Hum Neurosci 5:161

53. Lehky SR (1988) An astable multivibrator model of binocular rivalry. Perception 17:215-228

54. Oelkers-Ax R, Bender S, Just U, Pfuller U, Parzer P, Resch F, Weisbrod M (2004) Pattern-reversal visual-evoked potentials in children with migraine and other primary headache: evidence for maturation disorder? Pain 108:267-275

55. Conner IP, Sharma S, Lemieux SK, Mendola JD (2004) Retinotopic organization in children measured with fMRI. J Vis 4:509-523

56. Dougherty RF, Ben-Shachar M, Bammer R, Brewer AA, Wandell BA (2005) Functional organization of human occipital-callosal fiber tracts. Proc Natl Acad Sci USA 102:7350-7355

57. Saenz M, Fine I (2010) Topographic organization of V1 projections through the corpus callosum in humans. Neuroimage 52:1224-1229

58. Aboitiz F, Scheibel AB, Fisher RS, Zaidel E (1992) Fiber composition of the human corpus callosum. Brain Res 598:143-153

59. Innocenti GM, Price DJ (2005) Exuberance in the development of cortical networks. Nat Rev Neurosci 6:955-965

60. Pinto JG, Hornby KR, Jones DG, Murphy KM (2010) Developmental changes in GABAergic mechanisms in human visual cortex across the lifespan. Front Cell Neurosci 4:16

61. Eickhoff SB, Stephan KE, Mohlberg H, Grefkes C, Fink GR, Amunts K, Zilles K (2005) A new SPM toolbox for combining probabilistic cytoarchitectonic maps and functional imaging data. Neuroimage 25:1325-1335 